\title{
Optimization and nonlinear model predictive control of batch polymerization systems
}

\author{
Dulce C.M. Silva, Nuno M.C. Oliveira * \\ Departamento de Engenharia Química, Fac. de Ciências e Tecnologia, Universidade de Coimbra, Pólo II-Pinhal de Marrocos, \\ 3030-290 Coimbra, Portugal
}

\begin{abstract}
This paper addresses the optimization of batch polymerization systems, using a feasible path approach, with roots on Model Predictive Control (MPC) theory. The approach allows the reuse of many concepts previously developed for nonlinear MPC of continuous plants. It also provides an efficient and well-integrated methodology for the optimal supervision of discontinuous chemical processes. The application of this technique to the optimization of the batch suspension polymerization of vinyl chloride and the solution polymerization of methyl methacrylate is considered. Significant advantages associated to the use of this methodology are demonstrated with both examples, in terms of productivity gains and the capability of manufacturing products with pre-specified properties. (C) 2002 Elsevier Science Ltd. All rights reserved.
\end{abstract}

Keywords: Optimization; Polymerization; MPC

\section{Introduction}

Due to their nonstationary nature, batch processes present interesting challenges in their control and online optimization, and create unique opportunities for the development of advanced supervision strategies. In the case of batch polymerization processes, optimal operation involves computing and accurately maintaining the optimal temperature and initiator (or co-reactants) addition policies that can lead to a product with desired properties (such as molecular weight average, polidispersivity, chain length distribution) and final conversion, while minimizing the total operation time. In many cases, batch operations are still carried according to recipes based on heuristics and past experience. However, the recent availability of detailed mechanistic models, experimentally validated, provide a significant incentive for a wider use of newly developed optimization algorithms in batch systems operation.

Previous research on the determination of optimal policies for batch polymerization processes concentrated on techniques for the solution of optimization

\footnotetext{
* Corresponding author. Tel.: + 351-239-798700; fax: +351-239798703.

E-mail address: nuno@eq.uc.pt (N.M.C. Oliveira).
}

problems subject to algebraic and differential constraints. Several authors reported the use of the classical variational approach for the solution of specific problems in this field (Hicks, Mohan, \& Ray, 1969; Sacks, Lee, \& Biesenberger, 1972; Chen \& Jeng, 1978; Chen \& Huang, 1981; Thomas \& Kiparissides, 1984; Ponnuswamy, Shah, \& Kiparissides, 1987; Secchi, Lima, \& Pinto, 1990). To avoid the numerical difficulties associated with the solution of the resulting nonlinear two-point boundary value problem, various methods based on the discretization of the differential equations have been proposed (Cuthrell \& Biegler, 1987). These rely on weighted residuals, orthogonal collocation or finite differences schemes, using simultaneous solution of the differential equations and optimization. The application of these techniques to polymerization processes is considered by Jang and Yang (1989), Jang and Lin (1991), Crowley and Choi (1997, 1998), Gentric, Pla, Latifi, and Corriou (1999), Pinto and Giudici (2001).

On the other hand, nonlinear Model Predictive Control (MPC) algorithms using a feasible path approach have been tested with success on continuous processes, featuring highly nonlinear behaviour and open-loop instabilities, in the presence of general operating constraints (Oliveira \& Biegler 1995; Santos, Oliveira, \& 
Biegler, 1995). The method described in this paper implements a feasible path approach and is able to efficiently address a large number of optimization problems in this area. In this method the differential equations are solved in a separate phase from the optimization, allowing an accurate solution of the differential equations, by specialized numerical algorithms. The integration of the solution and optimization phases is performed similarly to the Newton Control formulation for nonlinear MPC of continuous chemical processes (Oliveira \& Biegler). As in the Newton control, the model and sensitivity equations are integrated along a candidate path. The values of the state variables are therefore obtained simultaneously with the input and initial condition sensitivities for a number of discrete time intervals covering the operating horizon. When these sensitivities become available, they are used for the construction of a dynamic matrix, containing first order information for the system, relative to the decision variables of the problem.

The formulation is sufficiently flexible to accommodate objectives such as the direct minimization of the operating time, or the deviations between a reference molecular weight distribution and the one obtained, subject to several constraints in the operating variables and specifications of the properties of the product, instantaneously and/or at the end of the run. An additional advantage of this approach is that if a Newton algorithm is used for online control, most of the program code and data structures can be reused between the optimization and control layers. The use of this methodology for general optimization of discontinuous processes is therefore investigated in this paper.

Section 2 describes the modifications required in the previously developed Newton Control formulation (Oliveira \& Biegler, 1995), to accommodate more general objectives in the optimization of discontinuous processes, such as the direct minimization of the operating time. Situations where the decision variables correspond to inputs or initial conditions of the problem are considered. Simulation results are presented in Sections 3 and 4. The application of this methodology to the batch suspension polymerization of vinyl chloride is considered in detail in Section 3. Two mechanistic models for this system are presented, allowing a comparison of the optimization results and their sensitivity to be established. A dynamical analysis of the system is also described together with the on-line implementation of the optimal trajectories obtained. Section 4 demonstrates the of use of this methodology to originate new products with prespecified final properties, in this case with the batch solution polymerization of methyl methacrylate (MMA).

\section{Problem formulation and solution strategy}

The dynamic optimization problems to be solved can be formulated as:

$$
\begin{aligned}
& \min _{\boldsymbol{u}(t) \in \mathscr{H}_{i k}} \\
& \quad \Psi(t, \boldsymbol{x}(t), \boldsymbol{u}(t)) \\
& \text { s.t. } \quad \dot{\boldsymbol{x}}=f_{p}(\boldsymbol{x}, \boldsymbol{u}) \\
& \boldsymbol{y}=g_{p}(\boldsymbol{x}) \\
& \boldsymbol{u}_{1} \leq \boldsymbol{u} \leq \boldsymbol{u}_{\mathrm{u}} \\
& \boldsymbol{x}_{1} \leq \boldsymbol{x} \leq \boldsymbol{x}_{\mathrm{u}} \\
& \boldsymbol{y}_{1} \leq \boldsymbol{y} \leq \boldsymbol{y}_{\mathrm{u}}
\end{aligned}
$$$$
\Psi(t, \boldsymbol{x}(t), \boldsymbol{u}(t))=G\left(\boldsymbol{x}\left(t_{\mathrm{F}}\right)\right)+\int_{t_{0}}^{t_{\mathrm{F}}} F(\boldsymbol{x}(t), \boldsymbol{u}(t), t) \mathrm{d} \tau
$$

where $f_{p}$ and $g_{p}$ are usually assumed continuous and differentiable, except perhaps at a finite number of switching points. Here $\boldsymbol{x} \in \mathbb{R}^{n_{\mathrm{s}}}$ is the state vector, $\boldsymbol{u} \in \mathbb{R}^{n_{i}}$ is the input vector, $\boldsymbol{y} \in \mathbb{R}^{n_{\mathrm{o}}}$ is the output vector, $\boldsymbol{u}_{1}, \boldsymbol{x}_{1}$ and $\boldsymbol{y}_{1}$ are lower bounds, while $\boldsymbol{u}_{\mathrm{u}}, \boldsymbol{x}_{\mathrm{u}}$ and $\boldsymbol{y}_{\mathrm{u}}$ are the corresponding upper bounds. $G\left(x\left(t_{\mathrm{F}}\right)\right)$ is a function of the state variables at the end of the run, and $F$ is a functional of the state and input variables along the operation path. Both are assumed to be general nonlinear, and twice differentiable.

This formulation is sufficiently general to express different objectives and constraints of various nature such as:

- The direct minimization of the operation time:

$$
\Psi(\cdot)=t_{\mathrm{F}}
$$

- The treatment of soft constraints, especially relative to the end of the operation, that can be formulated as:

$$
\Psi(\cdot)=\left(\boldsymbol{y}\left(t_{\mathrm{F}}\right)-\boldsymbol{y}_{\mathrm{sp}}\right)^{\mathrm{T}} \mathbf{Q}\left(\boldsymbol{y}\left(t_{\mathrm{F}}\right)-\boldsymbol{y}_{\mathrm{sp}}\right)
$$

where $\boldsymbol{y}_{\mathrm{sp}}$ represents the desired final values, $\boldsymbol{y}\left(t_{\mathrm{F}}\right)$ is the value of a set of output variables at the end of the run, and $\mathbf{Q}$ is a weighting matrix.

- Objectives related to regulation and tracking of an arbitrary trajectory, for a set of properties expressed in terms of the input, state and output variables, similarly to the nonlinear MPC strategy (Oliveira \& Biegler, 1995),

$$
\begin{aligned}
\Psi(\cdot)= & \int_{t_{k}}^{t_{k}+t_{\mathrm{oh}}}\left(\boldsymbol{y}-\boldsymbol{y}_{\mathrm{sp}}\right)^{\mathrm{T}} \mathbf{Q}_{\boldsymbol{y}}(t)\left(\boldsymbol{y}-\boldsymbol{y}_{\mathrm{sp}}\right) \\
& +\left(\boldsymbol{u}-\boldsymbol{u}_{r}\right)^{\mathrm{T}} \mathbf{Q}_{\boldsymbol{u}}(t)\left(\boldsymbol{u}-\boldsymbol{u}_{r}\right) \mathrm{d} \tau
\end{aligned}
$$

where $\boldsymbol{u}_{r} \in \mathbb{R}^{n_{\mathrm{i}}}$ and $\boldsymbol{y}_{\mathrm{sp}} \in \mathbb{R}^{n_{\mathrm{o}}}$ are reference trajectories for both the inputs and outputs. $\mathbf{Q}_{y} \in \mathbb{R}^{n_{\mathrm{o}} \times n_{\mathrm{o}}}$ and $\mathbf{Q}_{\boldsymbol{u}} \in \mathbb{R}^{n_{\mathrm{i}} \times n_{\mathrm{i}}}$ are adjustable weighting matrices in the objective function. The length of the output predic- 
tive horizon is given by $t_{\mathrm{oh}}$ and $t_{k}$ is the present sampling instant.

- Various rigid operational constraints written as algebraic or differential equalities and inequalities, that can be expressed in terms of the input, state and output variables of the model, in the form of Eqs. (2) and (3).

- Optimal initial conditions for the model Eqs. (2) and (3), e.g. optimal amounts of the initiators to be added at the beginning of the operation, according to the objective $\Psi(\cdot)$ chosen.

- Multiple simultaneous objectives, that can be reduced to a set of problems of the type above, by any of the available techniques.

The decision variables for this problem can be either input variables (e.g. temperature and initiator addition policies), or initial conditions for the state variables (e.g. quantities of each initiator to be added at the beginning of the operation).

The solution and implementation of the optimal poli-

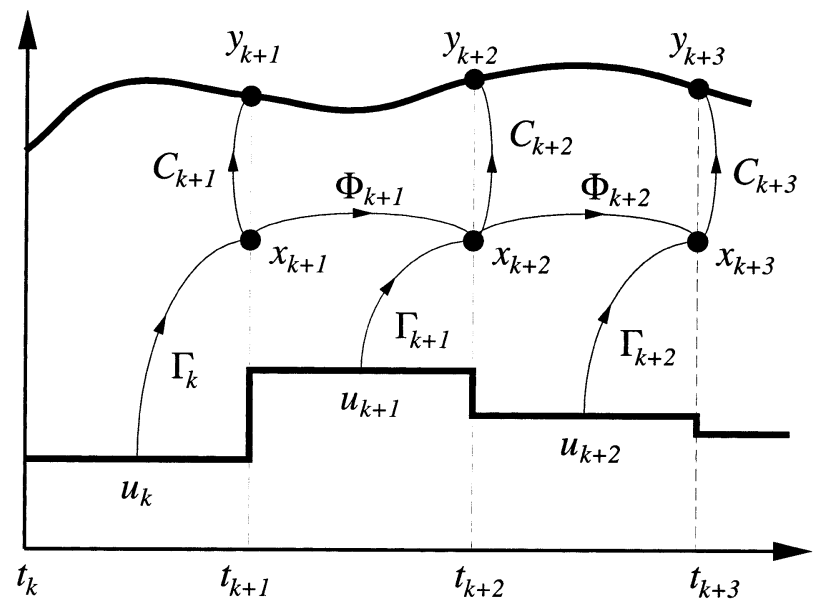

Fig. 1. Computing the input derivatives using state and input sensitivities.

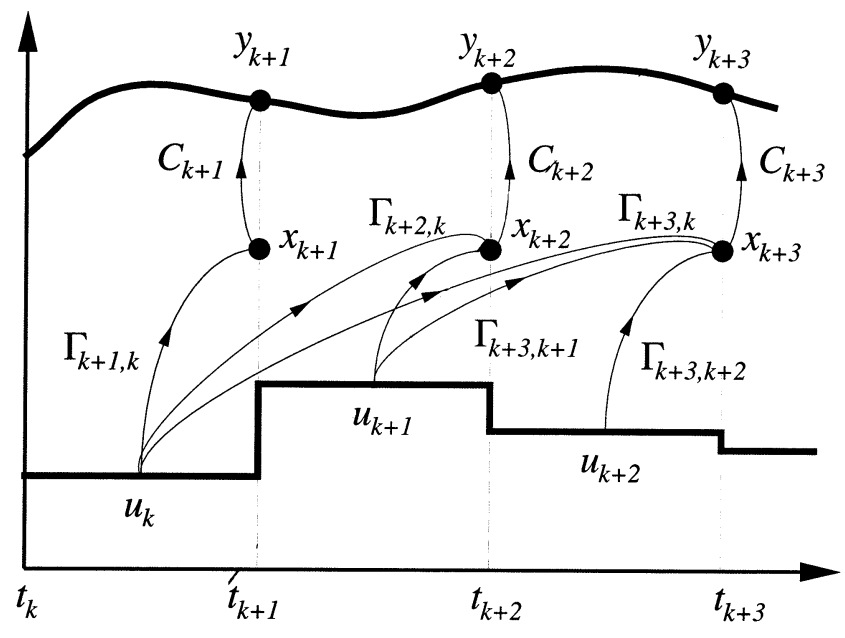

Fig. 2. Computing the input derivatives using only input sensitivities. cies is simplified by assuming piecewise constant input profiles $\boldsymbol{u}(t)$. The resulting nonlinear programming problem can be solved using a successive quadratic programming (SQP) approach, requiring the linear and quadratic terms of the constraints and objective. To simplify the notation, augmented vectors $\boldsymbol{U}, \boldsymbol{X}$ and $\boldsymbol{Y}$ are defined, containing all values of the corresponding variables inside an operating horizon:

$$
\begin{aligned}
\boldsymbol{U} \in \mathbb{R}^{n_{\mathrm{i}} m} & \equiv\left[\begin{array}{llll}
\boldsymbol{u}_{k}^{\mathrm{T}} & \boldsymbol{u}_{k+1}^{\mathrm{T}} & \cdots & \boldsymbol{u}_{k+m-1}^{\mathrm{T}}
\end{array}\right]^{\mathrm{T}} \\
\boldsymbol{X} \in \mathbb{R}^{n_{\mathrm{s}} p} & \equiv\left[\begin{array}{llll}
\boldsymbol{x}_{k+1}^{\mathrm{T}} & \boldsymbol{x}_{k+2}^{\mathrm{T}} & \cdots & \boldsymbol{x}_{k+p}^{\mathrm{T}}
\end{array}\right]^{\mathrm{T}} \\
\boldsymbol{Y} \in \mathbb{R}^{n_{\mathrm{o}} p} & \left.\equiv \begin{array}{llll}
\boldsymbol{y}_{k+1}^{\mathrm{T}} & \boldsymbol{y}_{k+2}^{\mathrm{T}} & \cdots & \boldsymbol{y}_{k+p}^{\mathrm{T}}
\end{array}\right]^{\mathrm{T}}
\end{aligned}
$$

Considering only input variables as decision variables, an exact linearization of the model around a nominal trajectory $\overline{\boldsymbol{U}}$, that represents an estimate of the solution, can be written as:

$\hat{\boldsymbol{Y}} \approx \overline{\boldsymbol{Y}}+\left.\frac{\partial \boldsymbol{Y}}{\partial \boldsymbol{U}}\right|_{\boldsymbol{U}=\overline{\boldsymbol{U}}} \Delta \boldsymbol{U}=\overline{\boldsymbol{Y}}+\mathscr{S}_{m} \Delta \boldsymbol{U}$

where $\Delta \boldsymbol{U}=\boldsymbol{U}-\overline{\boldsymbol{U}}$. The quantity $\mathscr{S}_{m}$ is the dynamic matrix of the model, containing the first order information for the system relative to its decision variables. This matrix can be efficiently computed from the original differential model through the use of appropriate sensitivity equations. The entries of the dynamic matrix can be expressed as:

$\mathscr{S}_{m_{i j}}=\left\{\begin{array}{cc}0 & \text { if } j>i \\ S_{i j} & \text { if } j<m \\ \sum_{l=j}^{i} S_{i l} & \text { otherwise }\end{array}\right.$

The terms $S_{i j}$ can be computed by different methods, either by partial derivation with respect to the state variables (Fig. 1), or by considering total derivatives with respect to the input variables (Fig. 2). In the first case one obtains:

$S_{i j}= \begin{cases}C_{k+i} \Gamma_{k+j-1} & \text { if } i=j \\ C_{k+i}\left(\prod_{m=1}^{i-j} \Phi_{k+i-m}\right) \Gamma_{k+j-1} & \text { otherwise }\end{cases}$

These sensitivity coefficients are defined by,

$\Phi_{k}=\frac{\partial \boldsymbol{x}_{k+1}}{\partial \boldsymbol{x}_{k}}, \quad \Gamma_{k}=\frac{\partial \boldsymbol{x}_{k+1}}{\partial \boldsymbol{u}_{k}}, \quad C_{k}=\left.\frac{\partial g(\boldsymbol{x})}{\partial \boldsymbol{x}}\right|_{\boldsymbol{x}=\boldsymbol{x}_{k}}$

Each sensitivity matrix can be obtained through the solution of the following system of differential equations (Oliveira, 1994):

$\frac{\partial \Phi\left(t, t_{k}\right)}{\partial t}=\left.\frac{\partial f_{p}(\boldsymbol{x}, \boldsymbol{u})}{\partial \boldsymbol{x}}\right|_{(\cdot)} \Phi\left(t, t_{k}\right)$

Initial conditions: $\Phi\left(t_{k}, t_{k}\right)=I_{n s}$. 


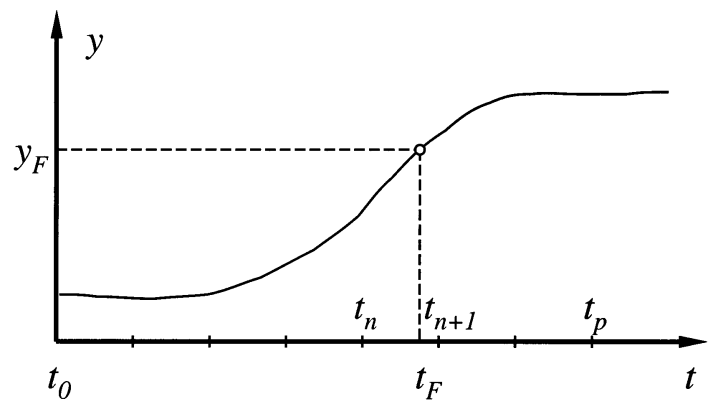

Fig. 3. Defining minimum time solutions.

$$
\begin{aligned}
\frac{\partial \Gamma\left(t, t_{k}\right)}{\partial t}= & \left.\frac{\partial f_{p}(\boldsymbol{x}, \boldsymbol{u})}{\partial \boldsymbol{x}}\right|_{(\cdot)} \Gamma\left(t, t_{k}\right) \\
& +\left.\frac{\partial f_{p}(\boldsymbol{x}, \boldsymbol{u})}{\partial \boldsymbol{u}}\right|_{(\cdot)}\left[\boldsymbol{U}\left(t-t_{k}\right)-\boldsymbol{U}\left(t-t_{k+1}\right)\right]
\end{aligned}
$$

Initial conditions: $\Gamma\left(t_{k}, t_{k}\right)=0$.

These equations can be solved together with Eq. (1) inside each corresponding sampling interval in the operating horizon. A total of $2 p$ sensitivity matrices, with $p n_{\mathrm{s}}\left(n_{\mathrm{s}}+n_{\mathrm{i}}\right)$ entries is required to compute $\mathscr{S}_{m}$ by this method. The second approach defines each term $S_{i j}$ as:

$S_{i j}=C_{k+i} \Gamma\left(t_{k+i}, t_{k+j-1}\right)$

This corresponds to the integration of only the input sensitivities during several sampling intervals where they are defined, resulting in a total of $m p-(m-1) m / 2$ coefficients of size $n_{\mathrm{s}} \times n_{\mathrm{i}}$. Since they originate similar information, the most adequate method can be chosen for each problem, taking into consideration the relative size of the input and state vectors.

This strategy can easily deal with model discontinuities at a finite number of points, usually associated with distinct operational phases, that occur frequently in batch processes. However, these will have to occur at the beginning (or end) of a sampling interval, whose length can simply be adapted to match their occurrence. The derivative information of the model, problem constraints and objective function allows us to formulate the dynamic optimization problem as a NLP, and use a SQP-type method for its solution (Oliveira \& Biegler, 1995; Santos et al., 1995). For some types of problems only the first order information of the objective is needed in a Gauss-Newton formulation, while for other cases the use of second order information of the Lagrangian in a full SQP-type method is beneficial.

\subsection{Solution of minimum time problems}

When the objective has the form of Eq. (8) or Eq. (9), the algorithms described in Oliveira and Biegler (1995); Santos et al. (1995) can be used directly. However, some modifications in this formulation are required to treat minimum time problems. In these problems, the final time is usually defined by a certain output variable, which reaches a predefined value $\boldsymbol{y}_{\mathrm{F}}=\boldsymbol{y}\left(t_{\mathrm{F}}\right)$ at the end of the operation (Fig. 3). The end point can be easily detected by modern integrators, when $\boldsymbol{y}_{\mathrm{F}}$ is specified as a limit value. We assume that this happens during the $n$th discretization interval, from $t_{n}$ to $t_{n+1}$, inside a larger horizon defined as a maximum bound on $t_{\mathrm{F}}$. Given the previous assumptions about the model, it is possible to write $t_{\mathrm{F}}$ as an implicit function of the initial conditions and input variables during this interval,

$t_{\mathrm{F}}=h\left(\boldsymbol{x}_{n}, \boldsymbol{u}_{n}\right)$

The first order information for $t_{\mathrm{F}}$ can then be obtained by writing a Taylor series in this interval:

$t_{\mathrm{F}}=\bar{t}_{\mathrm{F}}+\left.\frac{\partial t_{\mathrm{F}}}{\partial \boldsymbol{x}_{n}}\right|_{\boldsymbol{x}=\overline{\boldsymbol{x}}}\left(\boldsymbol{x}_{n}-\overline{\boldsymbol{x}}_{n}\right)+\left.\frac{\partial t_{\mathrm{F}}}{\partial \boldsymbol{u}_{n}}\right|_{\boldsymbol{u}=\overline{\boldsymbol{u}}}\left(\boldsymbol{u}_{n}-\overline{\boldsymbol{u}}_{n}\right)$

The derivatives $\left.\left(\partial t_{\mathrm{F}} / \partial \boldsymbol{x}_{n}\right)\right|_{\boldsymbol{x}=\overline{\boldsymbol{x}}}$ and $\left.\left(\partial t_{\mathrm{F}} / \partial \boldsymbol{u}_{n}\right)\right|_{\boldsymbol{u}=\overline{\boldsymbol{u}}}$ are, in some cases, difficult to obtain directly, by integration of the sensitivity coefficients, since Eq. (11) is usually not available in explicit form. However, since these coefficients are only needed for the last time interval, they can also be approximated by finite differences, without a significant penalty. Applying the previous concepts, the linearization of $t_{\mathrm{F}}$ with respect to the input variables can be written as:

$t_{\mathrm{F}}=\bar{t}_{\mathrm{F}}+S^{*} \Delta \boldsymbol{U}$

where

$S^{*} \in \mathbb{R}^{n_{i} p} \equiv\left[\begin{array}{llll}S_{n, 1}^{*} & S_{n, 2}^{*} & \cdots & S_{n, p}^{*}\end{array}\right]$

and

$S_{n, j}^{*}= \begin{cases}0 & \text { if } j>n \\ \left(\partial t_{\mathrm{F}} / \partial \boldsymbol{x}_{n}\right)\left(\prod_{m=1}^{n-j} \Phi_{n-m}\right) \Gamma_{j-1} & \text { if } j<n \\ \left.\left(\partial t_{\mathrm{F}} / \partial \boldsymbol{u}_{n}\right)\right|_{\boldsymbol{u}=\overline{\boldsymbol{u}}} & \text { if } j=n\end{cases}$

Constraints in the output variables can be imposed during the time profile (at each discretization instant) and/or at the end of the run, expressing either operating limits or specifications of the final product. It is therefore convenient to form two groups: output variables with restrictions along the operation $\left(\boldsymbol{Y}^{\mathrm{O}}\right)$ and output variables with restrictions at end of the run $\left(\boldsymbol{Y}^{\mathrm{F}}\right)$. Eq. (10) originates therefore,

$\hat{\boldsymbol{Y}}^{\mathrm{O}}=\overline{\boldsymbol{Y}}^{\mathrm{O}}+\mathscr{Y}_{m}^{\mathrm{O}} \Delta \boldsymbol{U}$

$\hat{\boldsymbol{Y}}^{\mathrm{F}}=\overline{\boldsymbol{Y}}^{\mathrm{F}}+\mathscr{S}_{m}^{\mathrm{F}} \Delta \boldsymbol{U}$

with $\mathscr{S}_{m}^{\mathrm{O}}$ and $\mathscr{S}_{m}^{\mathrm{F}}$ calculated in a similar form, using the corresponding $C_{k+i}$ output coefficients. This allows the solution of minimum time problems to be formulated as the SQP iteration of, 
$\min _{\Delta \boldsymbol{U}} J_{2}=\bar{t}_{\mathrm{F}}+S^{*} \Delta \boldsymbol{U}+\Delta \boldsymbol{U}^{\mathrm{T}} H \Delta \boldsymbol{U}$

s.t. $\quad \boldsymbol{U}_{1 d} \leq \Delta \boldsymbol{U} \leq \boldsymbol{U}_{\mathrm{u} d}$

$\boldsymbol{Y}_{1 d} \leq \mathscr{S}_{m}^{\mathrm{O}} \Delta \boldsymbol{U} \leq \boldsymbol{Y}_{\mathrm{u} d}$

$\mathscr{S}_{m_{n}}^{\mathrm{F}} \Delta \boldsymbol{U}=\Delta \boldsymbol{y}_{\mathrm{F}}$

where $\Delta \boldsymbol{y}_{\mathrm{F}}=\boldsymbol{y}_{\mathrm{sp}}-\boldsymbol{y}\left(t_{\mathrm{F}}\right)$, and $\mathscr{S}_{m_{n}}^{\mathrm{F}}$ is a submatrix of $\mathscr{S}_{m}^{\mathrm{F}}$, whose rows are the rows of $\mathscr{S}_{m}^{\mathrm{F}}{ }^{n}$ referring to the end of the run. $H$ represents an approximation of the Hessian of the Lagrangian of Eq. (1). This formulation is closely similar to the one used in the nonlinear Newton control law, making the algorithms developed for its solution applicable for minimum time problems as well.

\subsection{Optimization with respect to the initial conditions}

When initial conditions are present as decision variables in the optimization problem, a similar approach can be used to compute the derivatives of the model with respect to them. For example, a linearization of $t_{\mathrm{F}}$ is given by

$t_{\mathrm{F}}=\bar{t}_{\mathrm{F}}+S^{*} \Delta x_{\mathrm{o}}$

with $\Delta \boldsymbol{x}_{\mathrm{o}}=\boldsymbol{x}_{\mathrm{o}}-\overline{\boldsymbol{x}}_{\mathrm{o}}$, using a nominal initial condition $\overline{\boldsymbol{x}}_{\mathrm{o}}$ and first order information given by

$S^{*}=\frac{\partial t_{\mathrm{F}}}{\partial \boldsymbol{x}_{n}} \prod_{m=1}^{n} \Phi_{n-m}$.

Similarly, the model equations can be also written as

$\hat{\boldsymbol{Y}}=\overline{\boldsymbol{Y}}+\left.\frac{\partial \boldsymbol{Y}}{\partial \boldsymbol{x}_{\mathrm{o}}}\right|_{\boldsymbol{x}=\overline{\boldsymbol{x}}} \Delta \boldsymbol{x}_{\mathrm{o}}=\overline{\boldsymbol{Y}}+\mathscr{S}_{m} \Delta \boldsymbol{x}_{\mathrm{o}}$

with $\mathscr{S}_{m} \in \mathbb{R}^{\left(n_{\mathrm{o}} p\right) \times n_{\mathrm{o}}}$. In this case the row $i$ of the dynamic matrix can be expressed by

$\mathscr{S}_{m_{i}}=C_{i} \prod_{m=1}^{i} \Phi_{i-m}$.

Therefore, minimum time problems can be solved through the SQP iteration of

$\min J_{2}=\bar{t}_{\mathrm{F}}+S^{*} \Delta \boldsymbol{x}_{\mathrm{o}}+\Delta \boldsymbol{x}_{\mathrm{o}}^{\mathrm{T}} H \Delta \boldsymbol{x}_{\mathrm{o}}$

s.t. $\quad \boldsymbol{x}_{\mathrm{o}_{1 d}} \leq \Delta \boldsymbol{x}_{\mathrm{o}} \leq \boldsymbol{x}_{\mathrm{o}_{\mathrm{u} d}}$

$\boldsymbol{Y}_{1 d} \leq \mathscr{S}_{m}^{\mathrm{O}} \Delta \boldsymbol{x}_{\mathrm{o}} \leq \boldsymbol{Y}_{\mathrm{u} d}$

$\mathscr{S}_{m_{n}}^{\mathrm{F}} \Delta \boldsymbol{x}_{\mathrm{o}}=\Delta \boldsymbol{y}_{\mathrm{F}}$

Finally, when both the inputs and the initial conditions of the state variables are decision variables, we can write the decision vector as,

$$
\begin{aligned}
\boldsymbol{U}^{*} \in \mathbb{R}^{n_{\mathrm{i}} p+n_{\mathrm{s}}} & \equiv\left[\begin{array}{lllll}
\boldsymbol{u}_{k}^{\mathrm{T}} & \boldsymbol{u}_{k+1}^{\mathrm{T}} & \cdots & \boldsymbol{u}_{k+p-1}^{\mathrm{T}} & \boldsymbol{x}_{\mathrm{o}}^{\mathrm{T}}
\end{array}\right]^{\mathrm{T}} \\
& \equiv\left[\begin{array}{lll}
\boldsymbol{U}^{\mathrm{T}} & \boldsymbol{x}_{\mathrm{o}}^{\mathrm{T}}
\end{array}\right]^{\mathrm{T}}
\end{aligned}
$$

and formulate the minimum time problem as,

$\min _{\Delta \boldsymbol{U}, \Delta \boldsymbol{x}_{\mathrm{o}}} J_{2}=\bar{t}_{\mathrm{F}}+S^{*} \Delta \boldsymbol{U}^{*}+\Delta \boldsymbol{U}^{* \mathrm{~T}} H \Delta \boldsymbol{U}^{*}$ s.t. $\quad \Delta \boldsymbol{U}_{\mathrm{ld}}^{*} \leq \Delta \boldsymbol{U}^{*} \leq \Delta \boldsymbol{U}_{\mathrm{u} d}^{*}$

$\boldsymbol{Y}_{1 d} \leq \mathscr{S}_{m}^{\mathrm{O}} \Delta \boldsymbol{U}^{*} \leq \boldsymbol{Y}_{\mathrm{u} d}$

$\mathscr{S}_{m_{n}}^{\mathrm{F}} \Delta \boldsymbol{U}^{*}=\Delta \boldsymbol{y}_{\mathrm{F}}$

where the first order information of the objective function is given by,

$S^{*} \in \mathbb{R}^{n_{i} p+n_{\mathrm{s}}} \equiv\left[\begin{array}{lllll}S_{n, 1}^{*} & S_{n, 2}^{*} & \cdots & S_{n, p}^{*} & S_{n, p+1}^{*}\end{array}\right]$

and

$S_{n, j}^{*}= \begin{cases}0 & \text { if } n<j<p \\ \left(\partial t_{\mathrm{F}} / \partial \boldsymbol{x}_{n}\right)\left(\prod_{m=1}^{n-j} \Phi_{n-m}\right) \Gamma_{j-1} & \text { if } j<n \\ \left.\left(\partial t_{\mathrm{F}} / \partial \boldsymbol{u}_{n}\right)\right|_{u=\overline{\boldsymbol{u}}} & \text { if } j=n \\ \left(\partial t_{\mathrm{F}} / \partial \boldsymbol{x}_{n}\right) \prod_{m=1}^{n} \Phi_{n-m} & \text { if } j=p+1\end{cases}$

In this case, the linearized model equations can still be expressed by,

$\hat{\boldsymbol{Y}}=\overline{\boldsymbol{Y}}+\mathscr{S}_{m} \Delta \boldsymbol{U}^{*}$

with the entries of the dynamic matrix $\mathscr{S}_{m} \in \mathbb{R}^{\left(n_{\mathrm{o}} p\right) \times\left(n_{\mathrm{i}} p+\right.}$ $n_{\mathrm{s}}$ ) given by,

$$
S_{i j}= \begin{cases}C_{i} \Gamma_{j-1} & \text { if } i=j \\ 0 & \text { if } j>i \\ C_{i}\left(\prod_{m=1}^{i-j} \Phi_{i-m}\right) \Gamma_{j-1} & \text { if } j<i \\ C_{i} \prod_{m=1}^{i} \Phi_{i-m} & \text { if } j=p+1\end{cases}
$$

\section{Application to the vinyl chloride suspension polymerization}

In this section we consider the application of the previous strategy to the optimization and nonlinear control of the batch suspension polymerization of vinyl chloride (VCM). This system involves four phases (monomer, polymer, aqueous and gas), and a heterogeneous reaction. Various kinetic models have been proposed to describe the process, with significant differences at the level of complexity and detail given to various chemical and physical phenomena taking place.

In order to compare the optimization results, and to better assess their sensitivity, two mechanistic models for this process were built, based on the kinetics information provided by Xie, Hamielec, Wood, and Woods (1991), Kiparissides, Daskalatis, Achilias, and Sidiropoulou (1997). Both of these models consider diffusion controlled reactions. The monomer distribution in the different phases is computed as a function of the conversion and the reactor operation conditions. In Xie's 
model, the rate constants are modelled using the free volume theory, while in Kiparissides's model the termination and propagation rates are expressed in terms of reaction and diffusion limiting terms; the later term depends on an effective reaction radius and on the diffusion coefficients, calculated from the extended free volume theory (Kiparissides et al., 1997). The state variables introduced by Xie's model are the monomer conversion, the first and second moments of the dead polymer distribution in the polymer phase, number and weight accumulated molecular weight averages, and quantities of the initiator. In Kiparissides's model we have as state variables the conversion, zero, first and

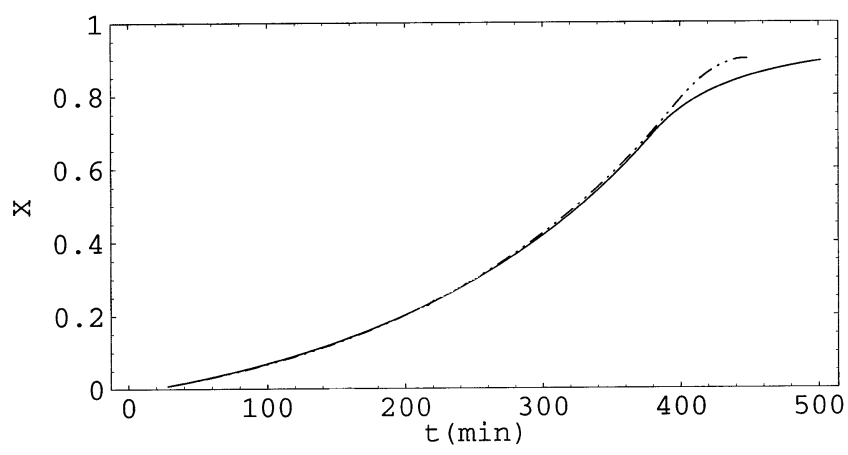

Fig. 4. Conversion profile for isothermal operation (-, Xie's model; -.., Kiparissides's model).

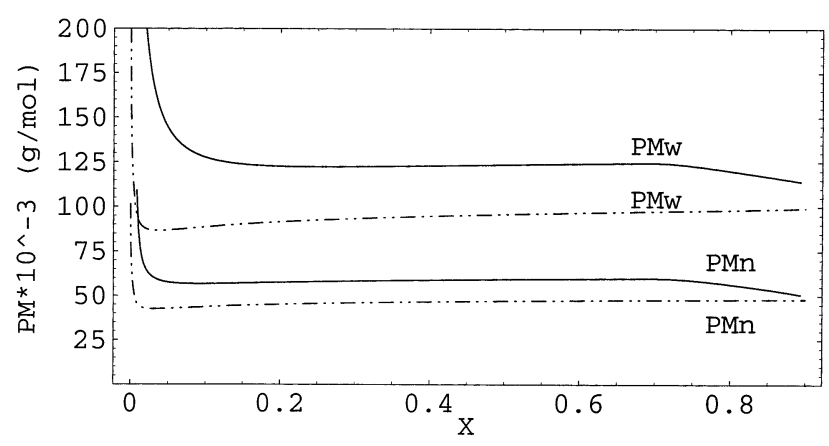

Fig. 5. Conversion dependence of number and weight average molecular weights (PMn and PMw, respectively) (_, Xie's model; —.., Kiparissides's model).

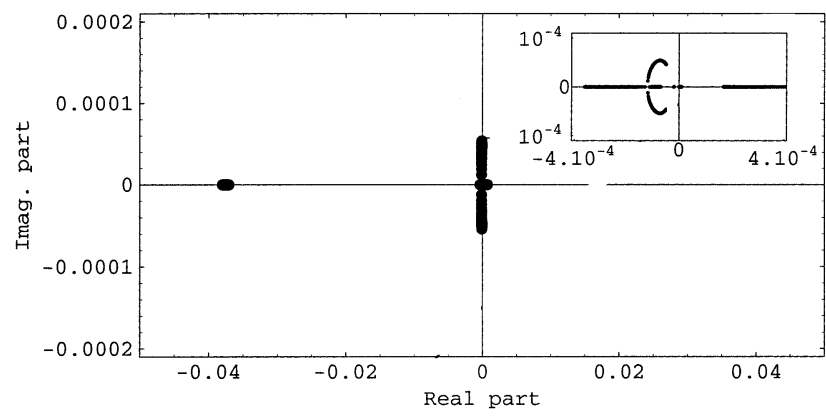

Fig. 6. Root locus of the linearized batch reactor, with a constant output profile, before the critical conversion is reached. second moments of the dead polymer distribution, and quantities of the initiators.

The predictions from both models are compared in Fig. 4, using a constant polymerization temperature of $55{ }^{\circ} \mathrm{C}$, with an equimolar mixture of initiators: (A) tert-amyl peroxyneodecanoate; and (B) tert-amyl peroxypivalate (Akzo Nobel Chemicals BV, 2000). As can be observed, the conversion profiles are close until a conversion of $70 \%$ is reached. Their divergence after this point can be attributed to the fact that in Kiparissides's model the initiation efficiency after the critical conversion is not considered diffusionaly controlled, as is in the Xie's model. With respect to average molecular weights, we can observe in Fig. 5 that for the same temperature the models predict polymers with slightly different properties at the end of the operation. These differences can be due to the values of the kinetic parameters used in each model, especially the chain transfer to monomer that controls the molecular weight of the polymer, as well as other considerations made in their development.

\subsection{Dynamic analysis of the model}

To develop a better understanding of the difficulties associated with the implementation of arbitrary optimal trajectories in this system, we studied the dynamic behaviour of a batch reactor of this type, including its jacket cooling system. This analysis was based on a constant polymerization temperature profile $(T=$ $55{ }^{\circ} \mathrm{C}$ ), which can be commonly found in current industrial practice. The complete dynamic model was linearized around this output reference trajectory, using the corresponding (variable) input reference profile. The eigenvalues of this model, using Kiparissides's kinetics information are plotted in Figs. 6 and 7. This dynamic model has a total of eight state variables (six state variables previously described, with energy balances for the reactor and jacket). Two distinct eigenvalue clusters are visible, before the critical conversion is reached. The first cluster, on the left, has only one negative eigenvalue, with position practically constant along the entire operation. The other cluster of dominant eigenvalues, located closer to the origin, has one null, three positive and three negative eigenvalues. In this group, two of the negative eigenvalues have an imaginary component. After the critical conversion (Fig. 7), two positive eigenvalues in this group become negative, with complex conjugated components. This analysis shows that the reactor is locally open-loop unstable around the nominal constant trajectory. Similar conclusions were obtained when the Xie's kinetic model was used with the same physical configuration (Silva, 2002). The state controllability matrix, obtained for the linearized model along the same operating points, displayed a numerical condition ranging from $10^{24}$ to $10^{32}$, 


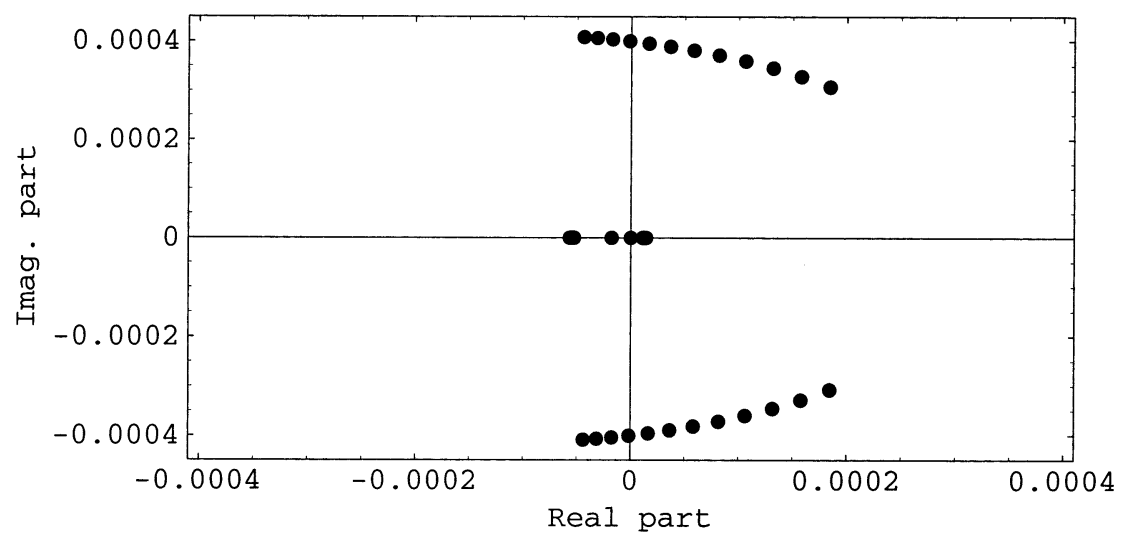

Fig. 7. A closer look at the root locus of the linearized model, after the critical conversion.

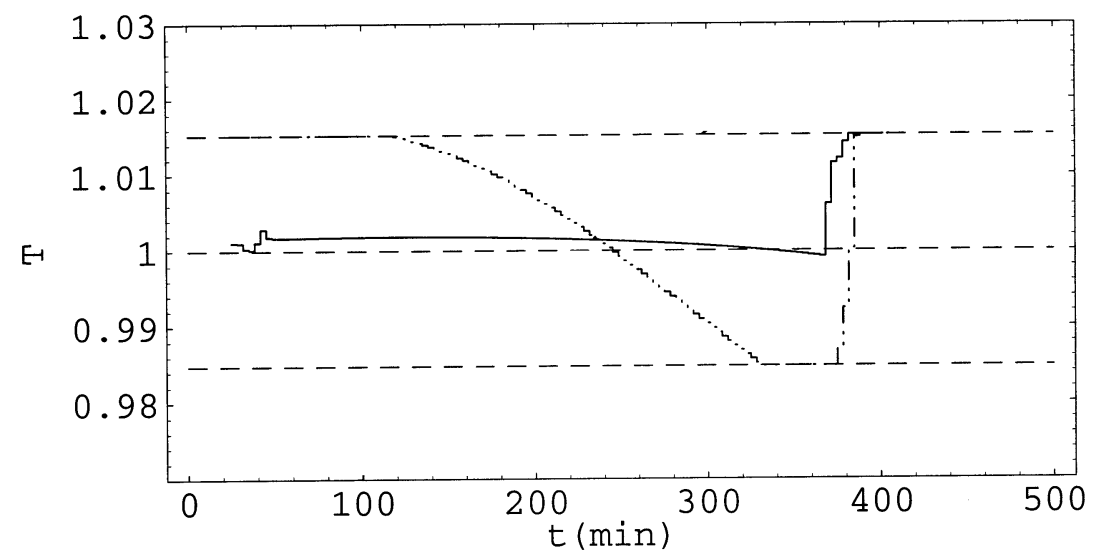

Fig. 8. Normalized optimal reactor temperature policy (—, Xie's model; —.., Kiparissides's model).

indicating that the arbitrary specification of all state variables can be difficult for this model (although not impossible).

\subsection{Optimization results}

Our optimization methodology was applied to the kinetic models described in Section 3.1, to determine the best operating profiles that lead to a product with desired properties in minimum time. Differently from the previous dynamic model, the reaction temperature and the concentrations of the initiators at the beginning of the operation were chosen here as main decision variables, in order to make the results applicable to reactors of different sizes and configurations. In this paper, we report only the effects of using each of these decision variables separately. Their combined effect is studied elsewhere (Silva, 2002).

Even when each decision variable is used separately, the occurrence of multiple local optima is possible, due to the general nonconvexity of the objective and the model equations. This depends on the particular nature of the objective function used and additional constraints that might be added to the formulation. The optimal profiles obtained in these examples were tested for the presence of multiple solutions by recomputing them from distinct initial estimates. No evidence of the existence of multiple local optima was found with the examples considered here.

\subsubsection{Effect of the reaction temperature}

Fig. 8 shows the results obtained for the optimal temperature profile that minimizes the batch time, when a product with identical properties to the polymer obtained using a constant temperature profile is desired (as described in Section 3.1). This is guaranteed by including in the formulation Eqs. (1)-(6) restrictions in the polidispersivity and molecular weight averages. Also, due to operational constraints, bounds of $\pm 5{ }^{\circ} \mathrm{C}$ relatively to the nominal temperature were imposed. As can be observed, when compared with isothermal operation, the profiles obtained with Kiparissides's and Xie's models are able to achieve reductions of 8.7 and $23 \%$ in the reaction time, respectively. Xie's model shows more sensitivity to the reaction temperature and therefore has lower deviations relatively to the nominal trajectory. After the critical conversion, the reaction temperature increases up to the maximum imposed, 
Table 1

Optimization of the initial quantities of the initiators

\begin{tabular}{llll}
\hline & Isothermal case & Xie's model \\
\hline Total amount (mol) & 18.0 & 19.4 & 53 \\
Initiator A $(\%)$ & 50 & 47 & 22.1 \\
Initiator B (\%) & 50 & 53 \\
Reduction in the cycle time $(\%)$ & & 47 \\
\hline
\end{tabular}

since in this phase of operation the majority of the polymer is already produced and this increase will not significantly affect the average properties of the polymer, while still contributing to reduce the total batch time.

\subsubsection{Effect of the initiator quantities}

The composition of the initiator, and the best amount that should be added at the beginning of the isothermal operation, in order to produce a polymer with desired properties (weight average molecular weight and polidispersivity) in minimum time, were also the subject of optimization; due to physical constraints, these quantities must be positive.

The results obtained are described in Table 1. As can be observed, both models also allow important reductions in the cycle time, compared to the base case, while suggesting a similar optimal initiator composition. The reduction obtained with the Xie model is smaller, because the initiation efficiency becomes diffusionaly controlled after the critical conversion.

\subsection{Control results}

The feasibility of implementing the optimal profiles previously determined was tested, using both a linear controller and a nonlinear Newton control strategy. A simple linear PI controller was selected, as representative of current industrial practice. Since the process was found to be open-loop unstable, the linear controller was tuned with the Ziegler-Nichols criteria, followed by manual retuning to avoid the appearance of oscillations at the beginning or end of the operation. Both the linear controller and the Newton predictive controller were initially tested with constant polymerization temperature profiles, and were able to effectively eliminate unknown disturbances, such as mismatches in the amount of initiators effectively added, or variations in the cooling flowrate and temperatures (Silva, 2002).

Here we consider their application in the implementation of the temperature profile that corresponds to the minimum time solution obtained using the dynamic model described in Section 3.1, and Kiparissides's kinetic information. This is illustrated in Figs. 9 and 10, using $k_{\mathrm{c}}=20$ and $\tau_{I}=1500 \mathrm{~s}$, with a sampling interval of $100 \mathrm{~s}$ for the linear controller, and $Q_{1}=I, Q_{2}=$
$10^{-3} I$ with a sampling time of $200 \mathrm{~s}$ for the Newton controller. To avoid the appearance of oscillations, while simultaneously providing good tracking properties, the linear controller needs to be carefully tuned for each reference profile, while the Newton controller is much less sensitive to the choice of adjustable parameters, and is able to operate with smaller amplitude changes in the manipulated variables and smaller response overshoots.

\subsection{Computational aspects}

The optimal operating profiles and the predictive control problems were solved using a prototype numerical implementation. This is composed by three modules, each corresponding to an independent process running on a Unix workstation. The modules for the simulation and solution of the quadratic optimization

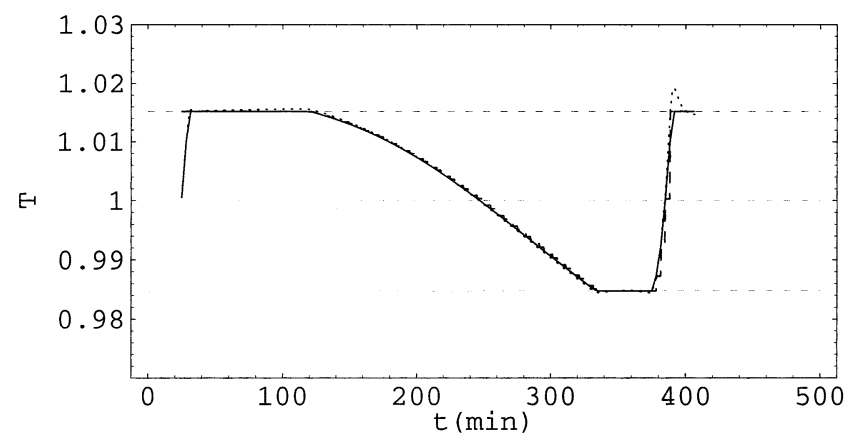

Fig. 9. Implementation of the optimal temperature policy for the PVC reactor (..., PI; - , MPC).

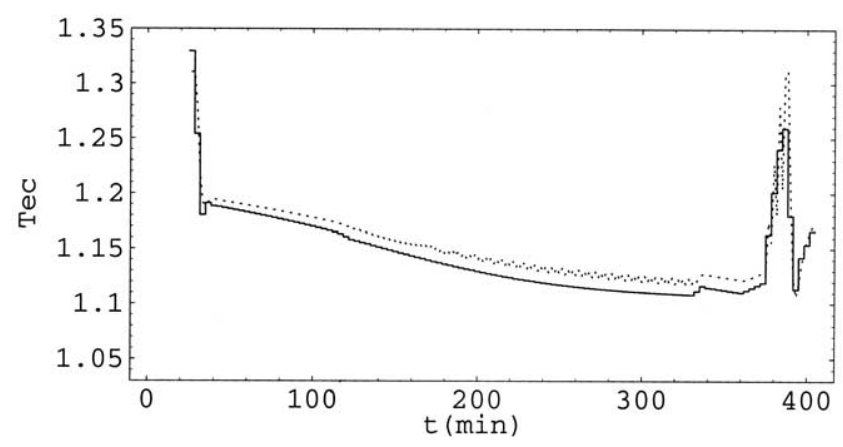

Fig. 10. Normalized inlet jacket temperature profile, for the optimal profile of Fig. 9 (..., PI; 一, MPC). 


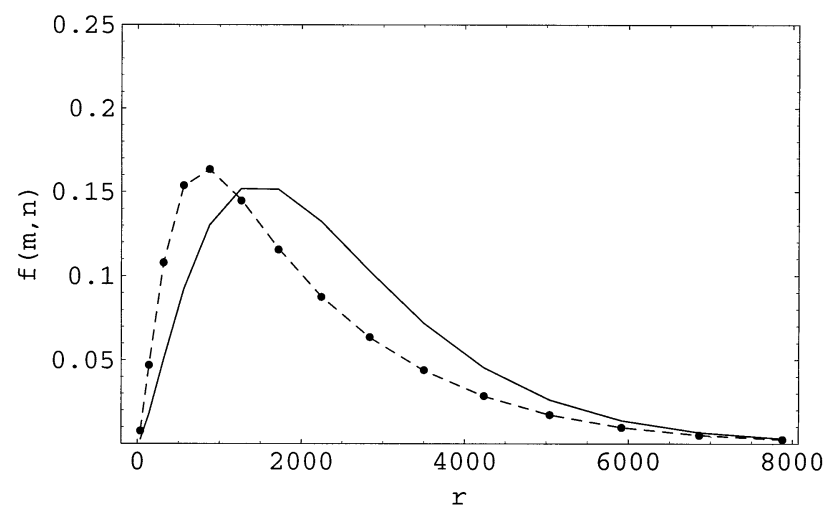

Fig. 11. Initial and final MMA polymer chain length distributions (--, desired; $\bullet$, obtained; - , initial).

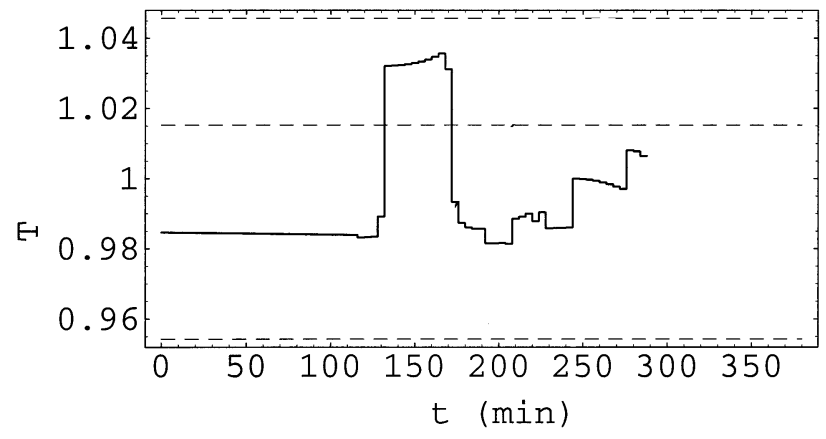

Fig. 12. Optimal MMA reactor temperature policy.

subproblems were written in Fortran, while the main part of the optimization algorithm itself was coded in the Mathematica language. To converge the optimal profiles within a tolerance of $5 \times 10^{-4}$, the number of iterations of the SQP algorithm ranged usually between 5 and 15. Typical CPU times for determining optimal profiles with Xie's model are $72 \mathrm{~min}$ for the model solution and sensitivity calculation, $2 \mathrm{~min}$ for the nonlinear optimization, and $6 \mathrm{~s}$ for the solution of the quadratic subproblems. The relatively high CPU times needed for the solution of the differential equations are in part due to the use of small integration stepsizes, in order to avoid jumps over large regions of operation, and the numerical difficulties associated. Further improvements in this efficiency of this phase are being considered presently.

\section{Application to the solution polymerization of MMA}

This section considers the application of the previous strategy to the optimization of the batch solution polymerization MMA, in order to demonstrate the ability of the method to originate products with pre-specified final properties.

The kinetics of free radical solution polymerization of MMA is relatively well known. The model used in this study is based on Crowley and Choi (1997) and includes mass balances on the monomer, polymer and solvent (ethyl acetate). Number and weight average molecular weights are calculated from the moments of the dead polymer molecular weight distribution. The state variables are monomer conversion $(X)$, weight fraction of polymer $\left(x_{\mathrm{p}}\right)$, weight fraction of solvent $\left(x_{\mathrm{s}}\right)$, zero, first and second order moments of the dead polymer molecular weight distribution $\left(\lambda_{0}, \lambda_{1}, \lambda_{2}\right)$, and $f(m, n)$ which corresponds to the weight fraction of polymer in the chain length interval from $m$ to $n$. In order to obtain a good compromise between the quality of the results and the dimension of the optimization problem, we used 15 intervals for the discretization of the chain length distribution, resulting in a model with 21 state variables.

The main goal for this example is the following: given that we are able to manufacture a product with the distribution represented by the solid line in Fig. 11 (e.g. using isothermal operation), we wish to find a temperature profile that enables us to produce a polymer with the chain length distribution represented by the dashed line in the same figure. A sampling interval of $4 \mathrm{~min}$, with horizons of length 90 was used in this case. We enforced a temperature constraint of $40<T<70{ }^{\circ} \mathrm{C}$. After some trials, the tuning parameters in the objective function were chosen as $Q_{1}=\{10, \ldots, 10,1,1,1,0.1$, $0.1,0.1\} I$, in order to produce a good fit of the desired response. Fig. 12 shows the optimal profile obtained. As can be observed from Fig. 11 the distribution obtained closely matches the desired one. After knowing that these product specifications are feasible, the direct minimization of the final time can be performed similarly to the previous example.

\section{Conclusions}

A nonlinear feasible path optimization strategy was considered in this paper, for the determination of optimal policies of batch processes. The formulation was demonstrated to be flexible, requiring only simple modifications in its basic structure in order to be able to address a large number of problems in this area.

This technique was illustrated with two batch polymerization systems. The suspension polymerization of vinyl chloride was considered by using two sources of kinetic information for this system, and comparing the optimal results predicted by these models. In each case, significant productivity improvements were demonstrated to be feasible, compared to the common industrial practice of isothermal operation. The implementation of the optimal solutions obtained can also be helpful in the validation of the currently available process models for this system. The online implementation of the optimal profiles obtained was 
considered, showing that the use of advanced control strategies, such as a similar Newton predictive formulation, can be advantageous in terms of better performance and flexibility in the choice of the tuning parameters.

Finally, an example of using this optimization approach to manufacture new products with pre-specified final properties was illustrated with the MMA system. These application examples clearly demonstrate the possible advantages that a more generalized use of these methodologies can bring to the area of batch systems operation.

\section{Acknowledgements}

The authors gratefully acknowledge the financial support provided by FCT under the scholarship PRAXIS/ $\mathrm{BD} / 9456 / 96$ and the research project 3/3.1/CEG/2577/95.

\section{References}

Akzo Nobel Chemicals BV (2000). Initiators for high polymers. Amersfoort, The Netherlands.

Chen, S.-A., \& Huang, N.-W. (1981). Minimum end time policies for batchwise radical chain polymerization-III. Chemical Engineering Science, 36, 1295-1305.

Chen, S.-A., \& Jeng, W.-F. (1978). Minimum end time policies for batchwise radical chain polymerization. Chemical Engineering Science, 33, 735-743.

Crowley, T. J., \& Choi, K. Y. (1997). Discrete optimal control of molecular weight distribution in a batch free radical polymerization process. Industrial and Engineering Chemistry Research, 36, $3676-3684$.

Crowley, T. J., \& Choi, K. Y. (1998). Experimental studies on optimal molecular weight distribution control in a batch-free radical polymerization process. Chemical Engineering Science, 53(15), 2769-2790.

Cuthrell, J. E., \& Biegler, L. T. (1987). On the optimization of differential-algebraic process systems. American Institute of Chemical Engineering Journal, 33(8), 1257-1270.

Gentric, C., Pla, F., Latifi, M. A., \& Corriou, J. P. (1999). Optimiza- tion and non-linear control of a batch emulsion polymerization reactor. Chemical Engineering Journal, 75(1), 31-46.

Hicks, J., Mohan, A., \& Ray, W. H. (1969). The optimal control of polymerization reactors. The Canadian Journal of Chemical Engineering, 47, 590-597.

Jang, S.-S., \& Lin, P.-H. (1991). Discontinuous minimum end-time temperature/initiator policies for batch emulsion polymerization of vinyl acetate. Chemical Engineering Science, 46(12), 31533163.

Jang, S.-S., \& Yang, W.-L. (1989). Dynamic optimization of batch emulsion polymerization of vinyl acetate - an orthogonal polynomial initiator policy. Chemical Engineering Science, 44(3), 515528.

Kiparissides, C., Daskalatis, G., Achilias, D. S., \& Sidiropoulou, E. (1997). Dynamic simulation of industrial poly(vinyl chloride) batch suspension polymerization reactors. Industrial and Engineering Chemistry Research, 36(4), 1253-1267.

Oliveira, N. M. C. (1994). Ph.D. Thesis. Carnegie Mellon University, USA.

Oliveira, N. M. C., \& Biegler, L. T. (1995). An extension of Newtontype algorithms for nonlinear process control. Automatica, 31, 281-286.

Pinto, J. M., \& Giudici, R. (2001). Optimization of a cocktail of initiators for suspension polymerization of vinyl chloride in batch reactors. Chemical Engineering Science, 56, 1021-1028.

Ponnuswamy, S. R., Shah, S. L., \& Kiparissides, C. A. (1987). Computer optimal control of batch polymerization reactors. Industrial and Engineering Chemistry Research, 26(11), 2229-2236.

Sacks, M. E., Lee, S., \& Biesenberger, J. A. (1972). Optimal policies for batch chain addition polymerizations. Chemical Engineering Science, 27, 2281-2289.

Santos, L. O., Oliveira, N. M. C., \& Biegler, L. T. (1995). Reliable and efficient optimization strategies for nonlinear model predictive control. Proceedings of dynamics and control of chemical reactors, distillation columns and batch processes (DYCORD + '95) (pp. 33-38).

Secchi, A. R., Lima, E. L., \& Pinto, J. C. (1990). Constrained optimal batch polymerization reactor control. Polymer Engineering and Science, 30(19), 1209-1219.

Silva, D. C. M. (2002). Ph.D. Thesis (in preparation). University of Coimbra, Portugal.

Thomas, I. M., \& Kiparissides, C. (1984). Computation of the near-optimal temperature policies for a batch polymerization reactor. The Canadian Journal of Chemical Engineering, 62, 284291.

Xie, T. Y., Hamielec, A. E., Wood, P. E., \& Woods, D. R. (1991). Experimental investigation of vinyl chloride polymerization at high conversion: mechanism, kinetics and modelling. Polymer, 32, 537-556. 